\title{
Glucosamine-induced ER Stress Accelerates Atherogenesis: A Potential Link between Diabetes and Cardiovascular Disease
}

\section{Vi T Dang, Daniel R Beriault, Arlinda Deng, Yuanyuan Shi and Geoff H Werstuck}

Thrombosis and Atherosclerosis Research Institute, McMaster University, Canada

"Corresponding author: Geoff H Werstuck, Thrombosis and Atherosclerosis Research Institute, McMaster University, Hamilton, Canada, Tel: 9055212100; Fax: 9055771427 E-mail: Geoff.Werstuck@taari.ca

Received date: November 25, 2015; Accepted date: December 24, 2015; Published date: December 27, 2015

Copyright: ( 2015 Dang VT, et al. This is an open-access article distributed under the terms of the Creative Commons Attribution License, which permits unrestricted use, distribution, and reproduction in any medium, provided the original author and source are credited.

\begin{abstract}
Background: Cardiovascular disease is the leading cause of death worldwide and is responsible for three out of four deaths in diabetic individuals. Our lack of understanding of the molecular mechanisms linking diabetes and atherosclerosis impedes the development of effective treatment strategies. Hyperglycemia and glucosaminesupplementation have been shown to induce endoplasmic reticulum (ER) stress and activate the unfolded protein response (UPR) in murine models of atherosclerosis. We hypothesize that diabetes/hyperglycemia promotes atherosclerosis by a mechanism involving glucosamine-induced ER stress/UPR activation and that attenuation of ER stress, using the chemical chaperone 4- phenylbutyric acid (4PBA), will slow the accelerated development of atherosclerosis.
\end{abstract}

Methods: Hyperglycemia was induced in female Apolipoprotein E-deficient (ApoE-/-) mice by multiple low-dose streptozotocin injections or by the introduction of the Ins2+/Akita mutation. Glucosamine-supplementation was achieved by adding different concentrations of glucosamine $(0.625-5 \% \mathrm{w} / \mathrm{v})$ to the drinking water of ApoE-/- mice. Subsets of mice from each group were also treated with 4PBA. The development of atherosclerosis was evaluated based on atherosclerotic lesion area and volume at the aortic sinus. Levels of protein O-linked $\mathrm{N}$-acetylglucosamine $(\mathrm{O}-\mathrm{GlcNAc})$ and ER stress markers were determined in atherosclerotic lesions using immunohistochemistry and immunofluorescence staining.

Results: Hyperglycemic and glucosamine-supplemented mouse models showed similar increases in O-GlcNAc and ER stress/UPR activation levels in atherosclerotic lesions. Lesion area was not significantly different between the three models of accelerated atherosclerosis. Glucosamine supplementation at $\geq 2.5 \%(\mathrm{w} / \mathrm{v})$ significantly increased lesional O-GIcNAc, UPR activation and atherosclerotic lesion area/volume, independent of changes in any measured metabolic parameters. 4PBA mitigated ER stress and attenuated accelerated atherosclerosis in both hyperglycemic and glucosamine-supplemented mouse models.

Conclusion: These findings suggest that hyperglycemia promotes accelerated atherosclerosis by a mechanism involving glucosamine-induced ER stress. Accelerated atherosclerosis can be attenuated in hyperglycemic ApoE-/mice by reducing ER stress levels.

Keywords: Atherosclerosis; Hyperglycemia; Glucosamine; ER stress

\section{Introduction}

Cardiovascular disease (CVD) is the leading cause of death worldwide [1]. Risk factors of CVD include obesity [2], dyslipidemia [3], smoking [4], hypertension [5] and diabetes mellitus [6]. Diabetes is a debilitating disease that affects millions of individuals and is increasing in prevalence at a dramatic rate $[7,8]$. Although CVD is responsible for approximately $75 \%$ of deaths in individuals with diabetes [9], the development of therapeutic strategies to prevent and/or treat CVD has been impeded due to the lack of understanding of the underlying biochemical mechanisms linking diabetes to CVD. Atherosclerosis is one of the major underlying causes of CVD, and expanding our knowledge of how diabetes promotes atherosclerosis, can facilitate the development of treatment strategies to slow or prevent the development of atherosclerosis.
All forms of diabetes are characterized, and clinically defined, by increased blood glucose levels, known as hyperglycemia. In an attempt to understand the pro-atherogenic effects of hyperglycemia, most of the research has been focussed on determining the pathways affected by the increase in glucose metabolism and the oxidative stress associated with the hyperglycemia $[10,11]$.

Although a number of pre-clinical studies supported the causative role of oxidative stress [12,13], antioxidant treatments have not demonstrated beneficial effects in reducing CVD risk in diabetic population in any of the large clinical trials in which they were tested $[14,15]$. This observation suggests that there are other causative mechanisms behind hyperglycemia-promoted atherosclerosis that may act independently, or work in parallel to, oxidative stress. Impaired endoplasmic reticulum (ER) function has been associated with the development of atherosclerosis in humans and in mouse models $[16,17]$. 
The ER is responsible for the folding, modification and trafficking of approximately one-third of all proteins produced in a typical eukaryotic cell [18]. When the influx of newly synthesized proteins exceeds the ER processing capacity, unfolded or misfolded proteins can accumulate and induce ER stress. The unfolded protein response (UPR) is a multifaceted, cellular self-defence mechanism that alleviates ER stress by attenuating de novo protein synthesis, increasing protein folding capacity and facilitating the degradation of irreversibly misfolded proteins $[18,19]$. Chronic ER stress/UPR activation has been associated with various pro-atherogenic processes including endothelial cell apoptosis, macrophage-foam cell inflammation and lipid accumulation [20].

The objective of this study is to investigate the role of glucosamineinduced ER stress/UPR activation in diabetic atherosclerosis. Previous research in our lab has shown that glucosamine-supplementation accelerates atherosclerosis in ApoE-/- mice [21,22]. We hypothesize that the concentration of glucosamine required to promote ER stress would be similar to that required to promote atherosclerosis. Furthermore, we hypothesize that reducing ER stress levels, using the chemical chaperon 4-phenylbutyric acid (4PBA), will attenuate the accelerated development of atherosclerosis in both hyperglycemic and glucosamine-supplemented mouse models.

\section{Materials and Methods}

\section{Animal models}

ApoE-/- mice were crossed with Ins2+/Akita mice to produce ApoE-/-:Ins2+/Akita offspring (n=4/group) [23]. Five-week-old female ApoE-/- mice were randomly divided into three groups ( $n=12 /$ group): control, 5\% glucosamine-supplemented and STZ-injected groups. Multiple low-doses ( $40 \mathrm{mg} / \mathrm{kg} /$ day for ten days) of STZ (SigmaAldrich) were injected intraperitoneally to induce hyperglycemia.

A different set of five-week-old female ApoE-/- mice were given regular drinking water $(\mathrm{n}=10)$, or water supplemented with $0.625 \%$ $(\mathrm{w} / \mathrm{v})(\mathrm{n}=4), 1.25 \%(\mathrm{n}=4), 2.5 \%(\mathrm{n}=6)$ or $5 \%(\mathrm{n}=9)$ glucosamine $(\mathrm{w} / \mathrm{v}$, Sigma-Aldrich) in drinking water. Subsets of control, $5 \%$ glucosaminesupplemented ApoE-/- and ApoE-/-Ins2+/Akita mice ( $\mathrm{n}=4$-5/group) were treated with $20 \mathrm{mM}$ 4PBA (Scandinavian Formulas Inc., PA) in drinking water.

This level of 4PBA has previously been shown to attenuate ER stress in mice [24]. All mice were given unrestricted access to water and standard chow diet (TD92078; Harlan Teklad, Madison, WI) and were maintained on a 12-hour light/dark cycle throughout the study. Triglycerides and cholesterol were measured using Infinity reagents (Thermo Scientific). All mice were sacrificed at 15 weeks of age, plasma and tissues were collected for analysis. All procedures were approved by McMaster University Animal Research Ethics Board.

\section{Histochemistry}

Formalin-fixed hearts were cut transversely and embedded in paraffin. Aortas including aortic root were sectioned, as previously described [25]. Serial sections $(5 \mu \mathrm{m})$ were stained with hematoxylin and eosin (Sigma-Aldrich) [25], or Masson's Trichrome (SigmaAldrich) to visualize atherosclerotic lesion area/volume.

Masson's Trichrome staining was performed based on SigmaAldrich's instruction in which nuclei, collagen and muscle/erythrocytes are stained in black, blue and red, respectively. Stained sections were imaged using a Leitz LABORLUX S microscope connected to a DP71 Olympus camera. Lesion area was quantified using Image J (1.48v) software. Lesion volume was computed as area under the curve of lesion area [25].

\section{Immunohistochemistry and immunofluorescence}

Paraffin imbedded serial sections were stained with the primary antibodies against KDEL (StressGen, Canada) for GRP78/GRP94, CTD110.6 (Convance Inc., CA) or RL2 (Affinity Bioreagent) for OGlcNAc, or GADD153 (Santa Cruz, CA) for CHOP, as previously described [25]. Negative controls were stained with IgG (SigmaAldrich) instead of primary antibodies to correct for non-specific staining. Stained sections were imaged using a Leitz LABORLUX S microscope connected to a DP71 Olympus camera. Positively stained area was quantified using Image J (1.48v) software.

\section{Plasma 4PBA quantification}

Plasma was extracted using an ice-cold mixture of 1:1 methanol:ethanol (v/v). L-phenylalanine- $\mathrm{d} 8 \quad(20 \mu \mathrm{M}$, Cambridge Isotope Laboratories, MA) was used as the internal standard. An Agilent $1200 \mathrm{RR}$ series liquid chromatography system (Agilent Technologies Inc., CA) coupled to a Bruker micrOTOF II (Bruker Daltonics, MA) mass analyzer equipped with an electrospray ionization (ESI) source (Agilent Technologies Inc.) was used to quantify plasma $4 \mathrm{PBA}$ concentration. A volume of $2 \mu \mathrm{L}$ of plasma extract was injected per run into a 2.1 x $50 \mathrm{~mm}$ Halo C8 column.

\section{Statistical analysis}

One-way ANOVA followed by Tukey's HSD test was used to compare results between multiple groups. Data were presented as the mean \pm standard deviation. For all experiments, $p$-value of $<0.05$ was considered statistically significant, ${ }^{*} \mathrm{p}<0.05,{ }^{* *} \mathrm{p}<0.01,{ }^{* *} \mathrm{p}<0.001$.

\section{Results}

\section{Hyperglycemia and glucosamine-supplementstion induce similar increases in vascular O-GlcNAc, ER stress and accelerated atherosclerosis in ApoE-/- mice}

Hyperglycemia was induced in female ApoE-/- mice by multiple low-dose STZ injections, or by the introduction of the Ins2Akita mutation. A separate group of ApoE-/- mice were supplemented with glucosamine $(5 \% \mathrm{w} / \mathrm{v})$ in the drinking water. At 10 weeks of age, STZinjected mice were severely hyperglycemic $(\mathrm{FBG}=26.9 \pm 2.1 \mathrm{mM})$, Ins2+/Akita mice were moderately hyperglycemic $(15.0 \pm 1.1 \mathrm{mM})$ and glucosamine-supplemented mice were normoglycemic $(7.1 \pm 0.3 \mathrm{mM})$, relative to ApoE-/- controls (7.4 $\pm 0.3 \mathrm{mM}$ ) (Figure 1A).

Plasma insulin levels of both hyperglycemic models were significantly lower than that of the control or glucosaminesupplemented ApoE-/- mice (Figure 1B). Up to 15 weeks of age, none of the mice showed any significant changes in plasma cholesterol or triglycerides (Figure 1C and 1D).

Consistent with previous findings, both hyperglycemia and glucosamine-supplementation significantly accelerated atherogenesis at the aortic sinus, relative to controls (Figure 2) [21-23]. Atherosclerotic lesion area was not significantly different between the three models of accelerated atherosclerosis. Protein O-linked N- 
acetylglucoamine (O-GlcNAc) levels have been directly correlated to intracellular levels of glucosamine [26].

We found that both hyperglycemic mouse models and the glucosamine-supplemented mouse model had similar elevations in the levels of lesional O-GlcNAc, relative to ApoE-/- controls (Figure 2). Glucosamine is a potent ER stress inducer in cultured vascular cells [22]. Both hyperglycemic and the glucosamine-supplemented mouse models showed similar increases in the levels of UPR proteins GRP78/94 and CHOP within the atherosclerotic lesions. Together these data are consistent with our hypothesis that hyperglycemia promotes atherosclerosis by a mechanism involving glucosamineinduced ER stress.
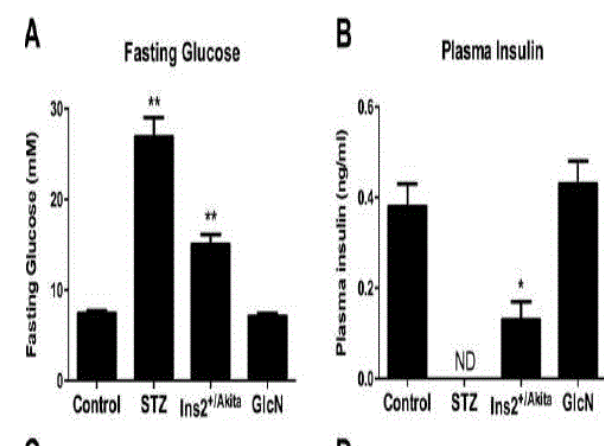

$$
\text { C }
$$
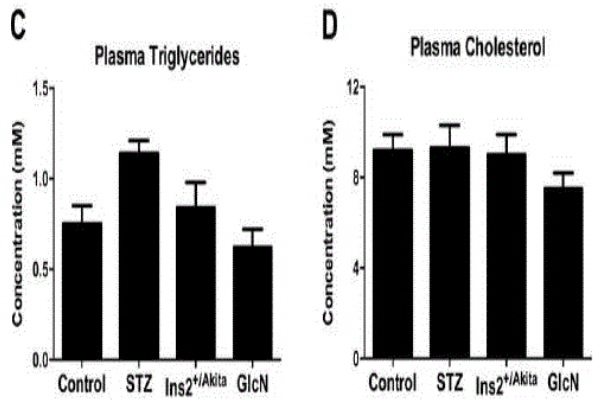

Figure 1: Analysis of metabolic parameters in three models of accelerated atherosclerosis. Fasting (A) blood glucose, (B) plasma insulin, (C) plasma triglycerides, and (D) plasma cholesterol levels of control, STZ-injected, Ins2+/Akita and glucosaminesupplemented ApoE-/- mice. ND indicates "not detected". $n=12 /$ group except for ApoE-/-Ins2+/Akita $\mathrm{n}=4$ /group, ${ }^{*} \mathrm{p}<0.05,{ }^{* *} \mathrm{p}<0.01$ relative to control mice

\section{Determining the glucosamine threshold level required to accelerate atherogenesis}

If ER stress is necessary to accelerate the development of atherosclerosis, then the concentration of glucosamine required to promote ER stress will be similar to that required to promote atherosclerosis. To determine this threshold level of glucosamine, fiveweek-old female ApoE-/- mice were supplemented with 0, 0.625, 1.25, 2.5 or $5 \%(\mathrm{w} / \mathrm{v})$ glucosamine in drinking water for 10 weeks. At 15 weeks of age, all mice were sacrificed and analysed. Glucosaminesupplementation did not alter any metabolic parameters including body weight, fasting glucose concentration, plasma cholesterol and triglycerides levels in ApoE-/- mice (Figure 3).

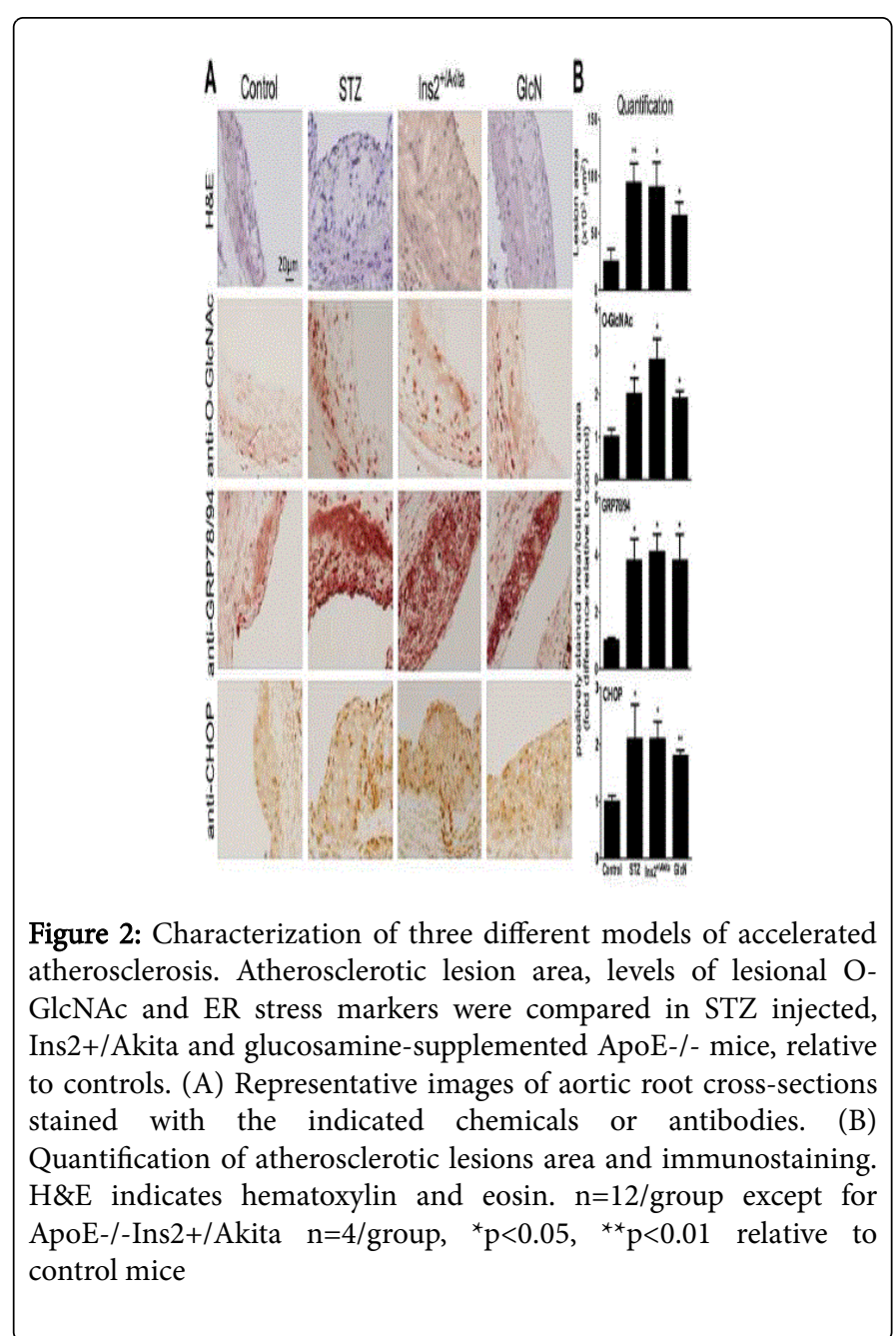

Supplementation at 0.625 and $1.25 \%(\mathrm{w} / \mathrm{v})$ did not significantly alter lesional protein linked O-GlcNAc (Figure 4A and Suppl. Figure 1) or UPR protein levels (Figure 4B-4C and Suppl. Figures 2, 3), nor did they affect atherosclerotic area or volume at the aortic sinus. Glucosaminesupplementation at 2.5 and $5 \%$ significantly increased lesional protein linked O-GlcNAc and also activated the UPR, which is indicative of ER stress in the atherosclerotic lesions (Figure 4).Furthermore, significantly larger atherosclerotic lesion area and volume were observed at the aortic sinus in mice supplemented with $\geq 2.5 \%$ glucosamine, relative to mice receiving $<2.5 \%$ glucosamine (Figure 5 ). These results are consistent with the hypothesis that glucosamineinduced ER stress promotes atherosclerosis.

\section{PBA mitigates ER stress and attenuates accelerated atherosclerosis}

We have identified a correlation between glucosamine-induced activation of the UPR and accelerated atherosclerosis. If elevated ER stress level is necessary for the accelerated development of atherosclerosis in glucosamine-supplemented mice then we would predict that the chemical chaperone, 4PBA, which attenuates ER stress, will also diminish the accelerated development of atherosclerosis in both glucosamine-supplemented and hyperglycemic mouse models. To test this, 4PBA was supplemented in drinking water of five week old 
Citation: Dang VT, Beriault DR, Deng A, Shi Y, Werstuck GH (2015) Glucosamine-induced ER Stress Accelerates Atherogenesis: A Potential Link between Diabetes and Cardiovascular Disease. J Mol Genet Med 9: 197. doi:10.4172/1747-0862.1000197

Page 4 of 7

ApoE-/- controls, glucosamine-supplemented ApoE-/- mice and ApoE-/-Ins+/Akita mice for 10 weeks.

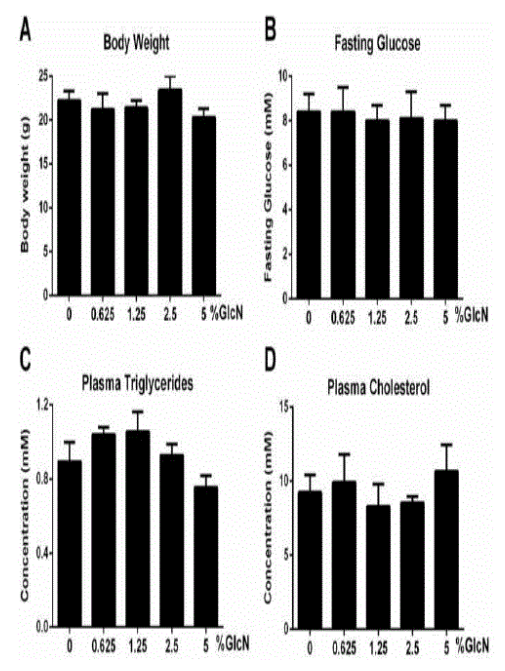

Figure 3: Analysis of metabolic parameters of mice supplemented with different levels of glucosamine in drinking water. (A) Body weight, fasting (B) blood glucose, (C) plasma triglycerides and (D) plasma cholesterol levels of control and $0.625,1.25,2.5$ and $5 \%$ (w/v) glucosamine (GlcN)-supplemented ApoE-/- mice. $n=4-6 /$ group

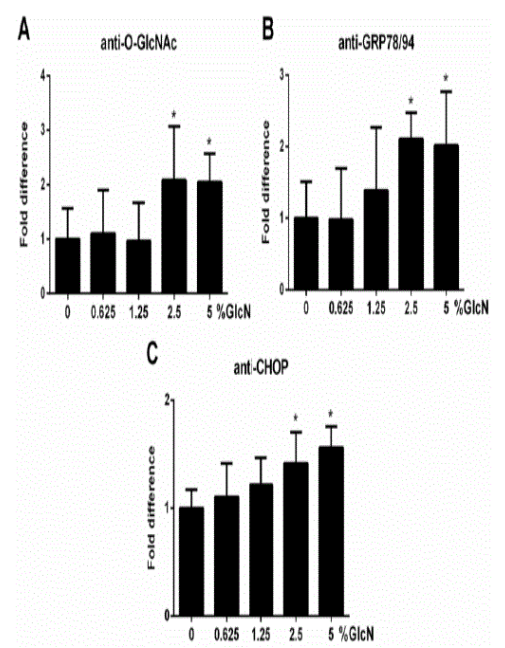

Figure 4: Comparison of O-GlcNAc and ER stress levels in atherosclerotic lesions of mice supplemented with different levels of glucosamine in drinking water. Quantification of (A) OGlcNAc, (B) GRP78 and (C) CHOP levels in aortic root cross-sections of the control and ApoE-/- mice supplemented with 0, 0.625, 1.25, 2.5 and $5 \%(\mathrm{w} / \mathrm{v})$ glucosamine $(\mathrm{GlcN})$. Positively stained areas were normalized to total lesion volume and presented as fold difference relative to control. $n=4-6 /$ group, ${ }^{\star} \mathrm{p}<0.05$ relative to control mice

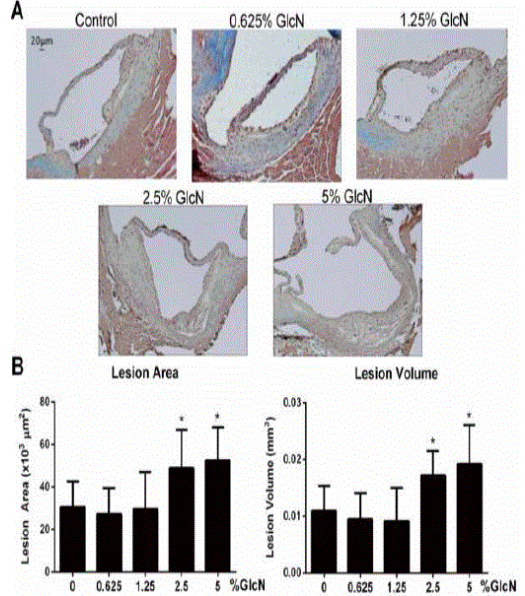

Figure 5: Determine the glucosamine threshold level required to accelerate atherogenesis. (A) Representative images of Masson's trichrome stained aortic cross-sections of mice supplemented with different concentrations of glucosamine. Quantification of atherosclerotic lesions (B) area and (C) volume. $n=4$-6/group, ${ }^{*} \mathrm{p}<0.05$

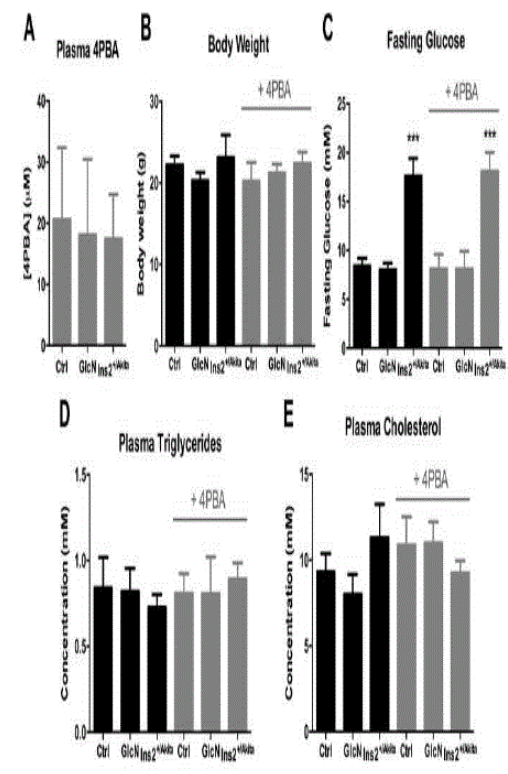

Figure 6: Analysis of metabolic parameters of hyperglycemic and glucosamine supplementation models in the absence or presence of 4BPA treatment. (A) Plasma 4PBA concentration of mice treated with 4PBA, (B) body weight, fasting (C) blood glucose, (D) plasma triglycerides and (E) plasma cholesterol levels of the control, 5\% glucosamine-supplemented ApoE-/- and ApoE-/- Ins2+/Akita mice with or without $4 \mathrm{PBA}$ treatment. $\mathrm{n}=4-6 /$ group, ${ }^{* *} \mathrm{p}<0.001$ relative to control mice

Plasma 4PBA concentration was measured using liquid chromatography coupled to a mass spectrometer. The results indicate that plasma 4PBA levels were not significantly different between any 
groups of mice treated with 4PBA (Figure 6A). Metabolic parameters including body weight, fasting glucose concentration, plasma cholesterol and triglycerides levels were not significantly different between mice with and without 4PBA treatment (Figure 6B-6E).Glucosamine-supplemented and hyperglycemic mice treated with 4PBA showed significant decrease in the levels of GRP78 and CHOP protein (Figure 7A, 7B and Suppl. Figures 4, 5), relative to mice without 4PBA treatment. 4PBA did not affect the level of ER stress indicators in the control mice. We found that $4 \mathrm{PBA}$ also reduced the levels of vascular O-GlcNAc in mice with 4PBA treatment, relative to those without $4 \mathrm{PBA}$ treatment, with the exception of control mice (Figure 7C and Suppl. Figure 6).

Atherosclerotic lesion area and volume were significantly decreased in glucosamine-supplemented ApoE-/- mice and ApoE-/-Ins+/Akita mice treated with $4 \mathrm{PBA}$, relative to mice without $4 \mathrm{PBA}$ treatment (Figure 8). 4PBA did not reduce atherosclerotic lesion area/volume in the control mice. Collectively, these results indicate that reducing ER stress levels attenuates the accelerated development of atherosclerosis.

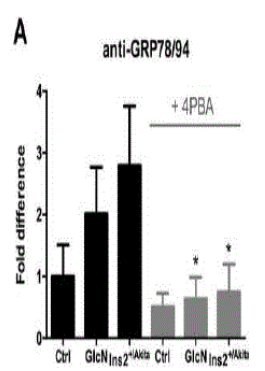

\section{B antichHop}

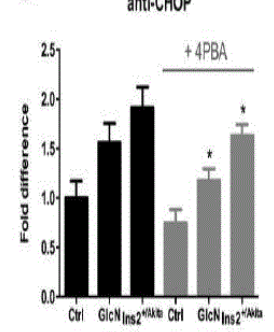

C

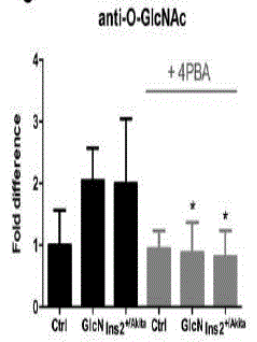

Figure 7: 4PBA reduces O-GlcNAc and ER stress levels in atherosclerotic lesions of glucosamine-supplemented and hyperglycemic mouse models. Quantification of (A) GRP78 and (B) $\mathrm{CHOP}$ and $(\mathrm{C}) \mathrm{O}-\mathrm{GlcNAc}$ levels in aortic cross-sections of the control, glucosaminesupplemented ApoE-/- and ApoE-/-Ins2+/ Akita mice with or without 4PBA supplementation. Positively stained areas were normalized to total lesion volume and presented as fold difference relative to control. $n=4-6 /$ group, ${ }^{*} \mathrm{p}<0.05$ relative to the same group of mice without $4 \mathrm{PBA}$ treatment

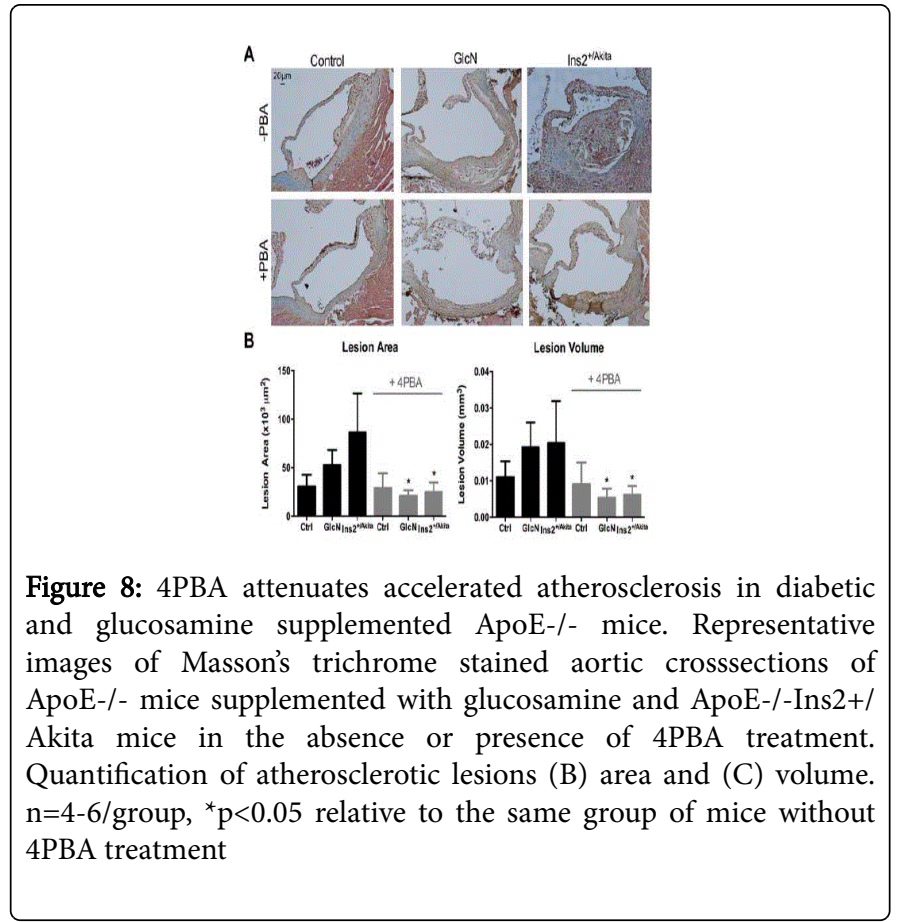

\section{Discussion}

In this study, we show that chemically (STZ injection) or genetically (Ins2+/Akita)-induced hyperglycemia promotes accelerated atherosclerosis in ApoE-/- mice in a manner that is independent of changes in plasma lipids. Similar increases in atherosclerotic lesion size were induced by supplementing ApoE-/- mice with glucosamine, which is a downstream metabolite of glucose. This study is the first direct comparison of these three mouse models of accelerated atherosclerosis. Each of these models showed a similar elevation in the level of O-GlcNAc and ER stress response proteins in atherosclerotic lesions, relative to ApoE-/- controls. In the titration experiments, we identified the threshold level of glucosamine required to induce ER stress is the same as that required to accelerate atherosclerosis $(\geq 2.5 \%$ $\mathrm{w} / \mathrm{v}$ in drinking water). Finally, we found that a chemical chaperone, which reduces ER stress levels in hyperglycemic and glucosaminesupplemented mice, attenuated the accelerated development of atherosclerosis. Together these findings are consistent with the mechanism in which hyperglycemia-associated atherogenesis is driven by glucosamine-induced ER stress.

The last few decades have witnessed a dramatic, worldwide increase in the prevalence of type 1 and especially type 2 diabetes that is likely driven by multiple environmental and lifestyle changes [7,8]. CVD is the leading cause of death in both type 1 and type 2 diabetes, even after controlling for other cardiovascular risk factors including dyslipidemia, obesity and hypertension [27,28]. All forms of diabetes are clinically defined by increased blood glucose concentration and there is a progressive relationship between hyperglycemia and CVD, with CV risk rising approximately $20 \%$ for every $1.5 \mathrm{mM}$ increase in fasting glucose levels [29], and for every $1 \%$ elevation in HbA1c levels [30]. Both epidemiological and pathophysiological studies have shown that hyperglycemia is an independent risk factor for CVD $[6,31,32]$. Hyperglycemia is known to increase flux through the HBP leading to increased production of UDP-N-acetylglucosamine (UDP-GlcNAc) - 
an essential substrate for both $\mathrm{N}$-linked and O-linked protein glycosylation $[33,34]$. Activation of the HBP has been implicated in the development of diabetic complications including glucotoxicity [35], insulin resistance [36], and cardiomyocyte dysfunction [37]. We have identified an additional consequence of enhanced HBP flux that involves the disruption of protein processing in the ER - a condition known as ER stress. Evidence from our lab and others has implicated ER stress in the development and progression of atherosclerosis in both humans and animal models [16,17]. Nonetheless, the mechanisms by which ER stress and/or UPR activation may promote the induction of pro-atherosclerotic pathways are not fully understood.

The chemical chaperone 4PBA has previously been shown to attenuate atherosclerosis in dyslipidemic mouse models [38]. However, this is the first demonstration that it can block accelerated atherogenesis associated with hyperglycemia. This observation is consistent with our hypothesis that ER stress plays a causative role in diabetes-associated atherosclerosis and further highlights the therapeutic potential of targeting the UPR as a viable strategy to treat and/or prevent atherosclerosis. The ability of glucosamine to induce ER stress is interesting because glucosamine is a popular dietary supplement used to treat joint pain associated with osteoarthritis [39]. To determine whether these supplements, when taken as directed (1500 mg/day), caused ER stress and/or activated the UPR in humans, we have previously measured markers of UPR activation in circulating peripheral blood cells isolated from fasting blood samples taken from volunteers before and after a 14 day regimen of glucosaminesupplementation. Our results suggested that this glucosaminesupplementation regimen does not result in UPR activation [40]. This is likely because this dose is insufficient to induce ER stress. Relative glucosamine concentrations in our diabetic and glucosaminesupplemented mouse models are estimated to be approximately 40 fold higher.

In conclusion, we have identified a molecular mechanism by which glucosamine-induced ER stress promotes the development of atherosclerosis. We propose that this mechanism may contribute to the accelerated atherosclerosis that is observed in mouse models of hyperglycemia and perhaps, in individuals with diabetes. Expanding the knowledge of how diabetes promotes atherosclerosis will facilitate in the development of novel, more effective therapeutic strategies to slow or stop atherogenesis.

\section{Acknowledgement}

This research was funded by operating grants from the Canadian Institutes of Health Sciences (MOP-142248) and the Canadian Diabetes Association (OG-3-12-3852-GW). The authors would like to thank the Centre for Microbial Chemical Biology at McMaster University for access to the Mass Spectrometer and Rostyslav Zvanych for critical reviewing of this manuscript.

\section{References}

1. Yusuf S, Hawken S, Ounpuu S, Dans T, Avezum A, et al. (2004) Effect of potentially modifiable risk factors associated with myocardial infarction in 52 countries (the INTERHEART study): case-control study. Lancet 364: 937-952.

2. Ozcan U, Cao Q, Yilmaz E, Lee A, Iwakoshi NN, et al. (2004) Endoplasmic reticulum stress links obesity, insulin action, and type 2 diabetes. Science 306: 457-461.

3. Karaskov E, Scott C, Zhang L, Teodoro T, Ravazzola M, et al. (2006) Chronic palmitate but not oleate exposure induces endoplasmic reticulum stress, which may contribute to INS-1 pancreatic beta-cell apoptosis. Endocrinology 147: 3398-3407.

4. Somborac-Bacura A, van der Toorn M, Franciosi L, Slebos D-J, ZanicGrubisic T, et al. (2013) Cigarette smoke induces endoplasmic reticulum stress response and proteasomal dysfunction in human alveolar epithelial cells. Exp Physiol 98: 316-325.

5. Young CN, Cao X, Guruju MR, Pierce JP, Morgan DA, et al. (2012) ER stress in the brain subfornical organ mediates angiotensin-dependent hypertension. J Clin Invest 122: 3960-3964.

6. Juutilainen A, Lehto S, Ronnemaa T, Pyorala K, Laakso M (2008) Similarity of the impact of type 1 and type 2 diabetes on cardiovascular mortality in middle-aged subjects. Diabetes Care 31: 714-719.

7. Hossain P, Kawar B, El Nahas M (2007) Obesity and diabetes in the developing world-a growing challenge. N Engl J Med 356: 213-215.

8. Patterson CC, Dahlquist GG, Gyürüs E, Green A, Soltész G (2009) Incidence trends for childhood type 1 diabetes in Europe during 1989-2003 and predicted new cases 2005-20: a multicentre prospective registration study. Lancet 373: 2027-2033.

9. Haffner SM, Lehto S, Ronnemaa T, Pyorala K, Laakso M (1998) Mortality from coronary heart disease in subjects with type 2 diabetes and in nondiabetic subjects with and without prior myocardial infarction. N Engl J Med 339: 229-234.

10. Brownlee M (2005) The pathobiology of diabetic complications: a unifying mechanism. Diabetes 54: 1615-1625.

11. Giacco F, Brownlee M (2010) Oxidative stress and diabetic complications. Circ Res 107: 1058-1070.

12. Brodsky SV, Gealekman O, Chen J, Zhang F, Togashi N, et al. (2004) Prevention and reversal of premature endothelial cell senescence and vasculopathy in obesity-induced diabetes by ebselen. Circ Res 94: 377-384.

13. Bucciarelli LG (2002) RAGE Blockade Stabilizes Established Atherosclerosis in Diabetic Apolipoprotein E-Null Mice. Circulation 106: 2827-2835.

14. Heart Outcomes Prevention Evalution Study Investigators (2000) Effects of ramipril on cardiovascular and microvascular outcomes in people with diabetes mellitus: results of the HOPE study and MICRO-HOPE substudy. Lancet 355: 253-259.

15. McQuillan BM, Hung J, Beilby JP, Nidorf M, Thompson PL (2001) Antioxidant vitamins and the risk of carotid atherosclerosis. The Perth carotid ultrasound disease assessment study (CUDAS). J Am Coll Cardiol 38: $1788-1784$

16. Minamino T, Komuro I, Kitakaze M (2010) Endoplasmic reticulum stress as a therapeutic target in cardiovascular disease. Circ Res 107: 1071-1082.

17. Tabas I (2010) The role of endoplasmic reticulum stress in the progression of atherosclerosis. Circ Res 107: 839-850.

18. Schröder M, Kaufman RJ (2005) ER stress and the unfolded protein response. Mutat Res 569: 29-63.

19. Pahl HL (1999) Signal transduction from the endoplasmic reticulum to the cell nucleus. Physiol Rev 79: 683-701.

20. Marciniak SJ, Ron D (2006) Endoplasmic reticulum stress signaling in disease. Physiol Rev 86: 1133-1149.

21. Beriault DR, Sharma S, Shi Y, Khan MI, Werstuck GH (2011) Glucosamine-supplementation promotes endoplasmic reticulum stress, hepatic steatosis and accelerated atherogenesis in apoE-/- mice. Atherosclerosis 219: 134-140.

22. Werstuck GH, Khan MI, Femia G, Kim AJ, Tedesco V, et al. (2006) Glucosamine-induced endoplasmic reticulum dysfunction is associated with accelerated atherosclerosis in a hyperglycemic mouse model. Diabetes 55: 93-101.

23. Venegas-Pino DE, Hong BY, Singh PN, Shi Y, Werstuck GH (2014) Gender specific differences in a new ApoE-/-:Ins2+/Akita mouse model of hyperglycemia-induced atherosclerosis. Canadian Journal of Cardiology 30: S325. 
Citation: Dang VT, Beriault DR, Deng A, Shi Y, Werstuck GH (2015) Glucosamine-induced ER Stress Accelerates Atherogenesis: A Potential Link between Diabetes and Cardiovascular Disease. J Mol Genet Med 9: 197. doi:10.4172/1747-0862.1000197

Page 7 of 7

24. Basseri S, Lhoták Š, Sharma AM, Austin RC (2009) The chemical chaperone 4-phenylbutyrate inhibits adipogenesis by modulating the unfolded protein response. J Lipid Res 50: 2486-2501.

25. Venegas-Pino DE, Banko N, Khan MI, Shi Y, Werstuck GH (2013) Quantitative analysis and characterization of atherosclerotic lesions in the murine aortic sinus. J Vis Exp 82: e50933.

26. Han I, Oh ES, Kudlow JE (2000) Resposiveness of the state of O-linked $\mathrm{N}$-acetylglucosamine modification of nuclear pore protein p62 to the extracellular glucose concentration. Biochem J 350: 109-114.

27. Lehto S, Rönnemaa T, Pyörälä K, Laakso M (2000) Cardiovascular risk factors clustering with endogenous hyperinsulinaemia predict death from coronary heart disease in patients with Type II diabetes. Diabetologia 43: 148-155.

28. Soedamah-Muthu S, Fuller J, Mulnier HE, Raleigh VS, Lawrenson R, et al. (2006) High risk of cardiovascular disease in patients with type 1 diabetes in the U.K.: a cohort study using the general practice research database. Diabetes Care 29: 798-804.

29. Seshasai SRK, Kaptoge S, Thompson A, Di Angelantonio E, Gao P, et al (2011) Diabetes mellitus, fasting glucose, and risk of cause-specific death. N Engl J Med 364: 829-841.

30. Selvin E, Steffes MW, Zhu H, Matsushita K, Wagenknecht L, et al. (2010) Glycated hemoglobin, diabetes, and cardiovascular risk in nondiabetic adults. N Engl J Med 362: 800-811.

31. Levitan EB, Song Y, Ford ES, Liu S (2005) Is nondiabetic hyperglycemia a risk factor for cardiovascular disease? A meta-analysis of prospective studies. Arch Intern Med 164: 2147-2155.

32. Zhang C-Y, Sun A-J, Zhang S-N, Wu C, Fu M-Q, et al. (2010) Effects of intensive glucose control on incidence of cardiovascular events in patients with type 2 diabetes: a meta-analysis. Ann Med 42: 305-315.
33. Denzel MS, Storm NJ, Gutschmidt A, Baddi R, Hinze Y, et al. (2014) Hexosamine pathway metabolites enhance protein quality control and prolong life. Cell 156: 1167-1178.

34. Helenius A, Aebi M (2004) Roles of N-linked glycans in the endoplasmic reticulum. Annu Rev Biochem 73: 1019-1049.

35. D’Alessandris C, Andreozzi F, Federici M, Cardellini M, Brunetti A, et al. (2004) Increased O-glycosylation of insulin signaling proteins results in their impaired activation and enhanced susceptibility to apoptosis in pancreatic beta-cells. FASEB J 18: 959-961.

36. Yang X, Ongusaha PP, Miles PD, Havstad JC, Zhang F, et al. (2008) Phosphoinositide signalling links O-GlcNAc transferase to insulin resistance. Nature 451: 964-969.

37. McNulty PH (2007) Hexosamine biosynthetic pathway flux and cardiomyopathy in type 2 diabetes mellitus. Focus on "Impact of type 2 diabetes and aging on cardiomyocyte function and O-linked $\mathrm{N}$ acetylglucosamine levels in the heart". Am J Physiol Cell Physiol 292: 1243-1244.

38. Erbay E, Babaev VR, Mayers JR, Makowski L, Charles KN, et al. (2009) Reducing endoplasmic reticulum stress through a macrophage lipid chaperone alleviates atherosclerosis. Nat Med 15: 1383-1391.

39. Clegg DO, Reda DJ, L HC, A KM, O’Dell JR, et al. (2006) Glucosamine, chondroitin sulfate, and the two in combination for painful knee osteoarthritis. N Engl J Med 354: 795-808.

40. Mcalpine CS, Beriault DR, Behdinan T, Shi Y, Werstuck GH (2014) Oral glucosamine sulfate supplementation does not induce endoplasmic reticulum stress or activate the unfolded protein response in circulating leukocytes of human subjects. Can J Physiol Pharmacol 92: 285-291. 